\title{
Подходы к управлению гибкостью системы высшего образования на основе учета социально-экономических особенностей регионов
}

\author{
Макарова Е.Л. \\ Южный федеральный университет \\ Россия, 344006, г. Ростов-на-Дону, ул. Большая Садовая, 105/42 \\ E-mail: elmakarova@sfedu.ru
}

\begin{abstract}
Аннотация. Методический подход к управлению отношениями «университет-бизнес-государство» по принципу «тройной спирали» обуславливает проведение исследования, определяющее и оценивающее эффективность взаимодействия высшего образования с субъектами экономики на региональном уровне и его роль в экономическом развитии региона. В ходе исследования автором были предложены рекомендации для разработки методики к реализации региональной политики по снижению асимметрии в региональных системах высшего образования и повышению их гибкости на основе учета социально-экономических особенностей регионов.
\end{abstract}

Ключевые слова: управление экономическими системами, тройная спираль, управление гибкостью, система высшего образования, социально-экономическая система, особенности регионов, региональная политика, сбалансированное развитие.

Благодарности: исследование выполнено при финансовой поддержке Российского фонда фундаментальных исследований (РФФИ), проект № 18-010-01115 «Разработка методологии и инструментария оценки эффективности функционирования региональной системы высшего образования и моделирование ее сбалансированного развития».

Для цитирования: Макарова Е.Л. 2021. Подходы к управлению гибкостью системы высшего образования на основе учета социально-экономических особенностей регионов. Экономика. Информатика, 48 (1): 17-24. DOI: 10.52575/2687-0932-2021-48-1-17-24.

\section{Approaches to managing the flexibility of the higher education system taking into account the socio-economic features of regions}

\author{
Elena L. Makarova \\ Southern Federal University \\ 105/42 Bolshaya Sadovaya St, Rostov-on-Don, 344006, Russia \\ E-mail: elmakarova@sfedu.ru
}

\begin{abstract}
As theoretical principles of management, the main aspects of substantiating the ideal or normatively balanced modeling of higher education institutions relationship, taking into account the needs of the region, were investigated and systematized in order to reduce the asymmetry in regional higher education systems and increase their flexibility based on taking into account the socio-economic characteristics of the regions. The study is devoted to the development of a methodology and tools for determining the socioeconomic efficiency for regional systems of higher education, modeling the balanced development of higher education institutions, taking into account the needs of the region and developing recommendations to improve their efficiency. During research, the author proposed guidelines for the development of methodical approaches to the implementation of regional policy to reduce asymmetry in regional higher education systems and increase their flexibility based on taking into account the socio-economic characteristics of the regions.
\end{abstract}

Keywords: management of economic systems, triple helix, flexibility management, higher education system, socio-economic system, peculiarities of regions, regional policy, balanced development. 
Acknowledgements: the research was supported within the grant provided by the Russian Foundation for Basic Research (RFBR), project no. 18-010-01115 "Development of methodology and tools for assessing the effectiveness of regional higher education system and modelling its balanced development".

For citation: Makarova E.L. 2021. Approaches to managing the flexibility of the higher education system taking into account the socio-economic features of regions. Economics. Information technologies, 48 (1): 17-24 (in Russian). DOI: 10.52575/2687-0932-2021-48-1-17-24.

\section{Введение}

В современном мире система высшего образования способствует региональному развитию тремя способами: во-первых, существует набор традиционно определенных преимуществ, таких как рост населения региона, увеличение спроса сферы услуг. Питание и жилье, повышение цен, увеличение расходов населения и другие сопутствующие социальноэкономические процессы [Фирсова, Нархова, 2014]. Во-вторых, есть неявные выгоды, широко связанные с ростом «экономики знаний» [Powel, Snellman, 2004], где прирост знаний региона включает в себя материальные (количественные) и нематериальные (качественные) преимущества. Такого рода выгоды связаны с обучением и передачей знаний и профессиональной подготовкой высококвалифицированных специалистов, которые могут происходить в регионе [Федоляк, 2018]. В-третьих, поскольку такие концепции, как «обучающиеся» регионы [Morgan, 1997], предлагают гибкие и инновационные меры реагирования на быстро меняющуюся экономику, существует предположение, что система высшего образования прямо или косвенно становится частью системы взаимосвязи институтов высшего образования с учетом потребностей региона [Fonseca et al., 2020], необходимых для стимулирования такого рода образовательных программ. Эти преимущества потенциально позволяют университетам вносить не только большой вклад в развитие своего региона, но и стратегически позиционировать этот регион как передовой в «экономике знаний».

Методический подход, изложенный в этом исследовании, разрабатывает основу для создания методологии и инструментария при определении социально-экономической эффективности региональных систем высшего образования, а также моделирования сбалансированного развития институтов высшего образования с учетом потребностей региона по принципу «тройной спирали» [Etzkowitz, 2008]. Данный методический подход нацелен на разработку рекомендаций по повышению эффективности системы высшего образования обучающегося региона к экономическому развитию, что обеспечивает динамическую основу для стимулирования инноваций и развития предпринимательства, как отличительного признака экономической устойчивости в постиндустриальной экономике.

\section{Методология исследования}

При разработке рекомендаций по повышению эффективности функционирования региональных систем высшего образования и управлению гибкостью на основе учета социально-экономических особенностей регионов необходимо учитывать пять основных аспектов обоснования идеальной или нормативно сбалансированной модели взаимосвязи институтов высшего образования с учетом потребностей региона.

Теоретическое ядро модели взаимосвязи институтов высшего образования с ключевыми субъектами в регионе включает в себя рассмотрение поведения сложных социальноэкономических систем, анализа динамики сбалансированного развития данных триад, разработки и обоснования управленческих решений для обеспечения устойчивого социальноэкономического развития региона на основании принципа необходимости. При моделировании взаимосвязи институтов высшего образования с учетом потребностей региона в элемент модели также привносят склонность к инновациям, особенно в области организационных инноваций и изобретения новых организационных форматов. Модель взаимосвязи институтов высшего образования с учетом потребностей региона в своей первоначальной разработке фокусируется на взаимных отношениях / взаимодействиях между тремя субъек- 
тами: системой высшего образования, социальной и экономической системами региона [Ogurtsova et al., 2018].

Основной механизм, лежащий в основе взаимодействия модели взаимосвязи институтов высшего образования с ключевыми субъектами в регионе, как оптимального условия по снижению асимметрии в региональных системах высшего образования и повышению их гибкости на основе учета социально-экономических особенностей регионов, предполагает выполнение субъектами взаимодействия новых функций наряду с традиционными. Учреждения, выполняющие нетрадиционные функции, рассматриваются, как главный потенциальный источник инноваций в регионе. Например, субъекты бизнессообщества продолжают производить товары и услуги, а также финансируют научноисследовательскую деятельность и проводят непрерывное обучение своих специалистов. Субъекты государственного управления несут ответственность за устранение сбоев рыночного механизма региона, корректировку государственной политики и установление рыночных правил региона, а также предоставляют венчурный капитал для финансирования предприятий инновационного сектора, особенно для предприятий с высоким уровнем риска. Университеты сохраняют свою традиционную роль в обучении и проведении исследований, а также прилагают усилия для капитализации знаний, патентов и создания инновационных предприятий региона [Мурашова и др., 2016]. В действительности все большее число университетов развивают сложную инновационную систему, начиная с офисов передачи технологий, бизнес-инкубаторов и научных парков, заканчивая международными исследованиями и обучению основам ведения бизнеса на территории кампуса. Реализация современными университетами образовательной, научно-исследовательской и социальной функций становится значимой составляющей экономического роста региона. В исследовании были выявлены количественные показатели, позволяющие оценить функциональную структуру и динамику систем высшего образования региона при выполнении образовательной и научной функций, включая региональное партнерство. Показано, что общая эффективность функционирования социально-экономической системы состоит из двух элементов: 1) эффективности функционирования каждой подсистемы; 2) степени согласованности подсистем [Огурцова, Челнокова, 2018]. Определение состояния региональных систем высшего образования было представлено с помощью показателя эффективности на основе резервов (SBM), который является вариантом анализа охвата данных (DEA) [Фирсова, Чернышова, 2019]. Данный метод оболочечного анализа данных позволяет строить границу эффективности выборки регионов, оценивая региональную систему высшего образования. Полученный рейтинг дает возможность объективного принятия решений по повышению эффективности функционирования региональных систем высшего образования и повышению их гибкости на основе учета социальноэкономических особенностей регионов.

Эволюционные механизмы лежат в основе развития модели «тройной спирали». Однако модели взаимосвязи институтов высшего образования с ключевыми субъектами в регионе не является результатом самоорганизованной эволюции; скорее, процесс разработки должен быть предварительно структурирован и скоординирован с учетом региональной инновационной политики [Cai, Liu, 2020]. Согласно исследованиям Ю. Каи и Г. Ицковица [Cai, Etzkowitz, 2020], замечено, что спирально развивающаяся модель взаимосвязи институтов высшего образования с ключевыми субъектами в регионе представляет собой «синтез эволюции по вертикальной оси и циркуляции по горизонтали» [Etzkowitz, 2008]. Выполнение новых функций субъектами взаимодействия в основном отражает горизонтальную циркуляцию с точки зрения наблюдаемых действий, оно приводит к эволюции каждой из трех систем (региональная система высшего образования, социальная и экономическая системы) по вертикальной оси и модели их взаимодействия. Г. Ицковиц [Etzkowitz, Leydesdorff, 2000] и Л. Лейдесдорф [Leidesdorff, 2012], соответственно, используют неоинституциональную теорию и неоэволюционную теорию при понимании эволюционных механизмов. С точки зрения институциональной теории, поскольку среда, то есть модели вза- 
имодействий тройной спирали между субъектами системы высшего образования, бизнессообществом и органами государственной власти на региональном или государственном уровне, могут со временем изменяться, сущность организации трех секторов может трансформироваться вместе с изменениями окружающей среды. С эволюционной точки зрения три функции (экономическое состояние, производство знаний и нормативный контроль) являются тремя эволюционными механизмами.

Диагностика состояния конгруэнтности взаимосвязи субъектов региональной системы высшего образования и социально-экономической системой и моделирование сбалансированного развития «тройной спирали» с учетом потребностей региона заключается в том, чтобы задействовать функциональные механизмы управления, посредничающие между нисходящими и восходящими инициативами. Роль законодательной власти заключается в разработке инновационной политики и инициировании приоритетных инновационных программ на региональном уровне. Однако динамика модели также опирается на инициативы снизувверх, которые сочетаются с «активным гражданским обществом, в котором инициативы поощряются различными частями общества» [Etzkowitz, 2008]. При проведении исследования была обоснована процедура диагностики сбалансированного развития региональных систем высшего образования с учетом потребностей региона с помощью когнитивного моделирования, включая разработку когнитивной модели; исследование свойств сложной слабоструктурированной системы; разработку сценарного анализа; и корректировку когнитивной модели выбранного региона [Макарова, 2018].

Для создания оптимальных условий по повышению эффективности функционирования региональных систем высшего образования необходима активация лидерства со стороны индивидуумов и организаций, пользующихся уважением всех ключевых игроков региона. Также инновационный потенциал как необходимое условие заключается в наличии и способности создать базу знаний с потенциалом коммерциализации. Это может быть подтверждено традиционными показателями исследований и разработок, такими как расходы бизнес-предприятий на НИОКР и валовые внутренние расходы на исследования и разработки, дополненные зарегистрированными патентами, доступом к информации и другими показателями инновационного потенциала базы знаний региона [Cai, Etzkowitz, 2020].

\section{Основные результаты исследования}

Результаты исследования позволяют предложить направления совершенствования и повышения эффективности системы высшего образования с целью снижения асимметрии в региональных системах и повышения их гибкости на основе учета социально-экономических особенностей регионов [Сучков, Ревин 2012; Иванова, 2012]. Конгруэнтность взаимосвязи субъектов региональной системы высшего образования и социально-экономической системы включает несколько направлений (рисунок).

Управление гибкостью позволит руководству высшего учебного заведения генерировать и реализовывать инновационные проекты, коммерциализировать новые идеи, фокусирующиеся на повышении эффективности деятельности социально-экономической системы региона. Управление гибкостью региональной системы высшего образования характеризует способность руководства к быстрой перестройке управленческого воздействия всей его системы, принятию управленческих решений и быстрая адаптация к сложившимся социальноэкономическим обстоятельствам ради поддержания инновационной активности высшего учебного заведения и наращивания конкурентоспособности региона с целью создания сбалансированной системы взаимосвязи институтов высшего образования с учетом потребностей региона с целью направления снижения асимметрии и управления гибкостью на основе учета социально-экономических особенностей регионов. 


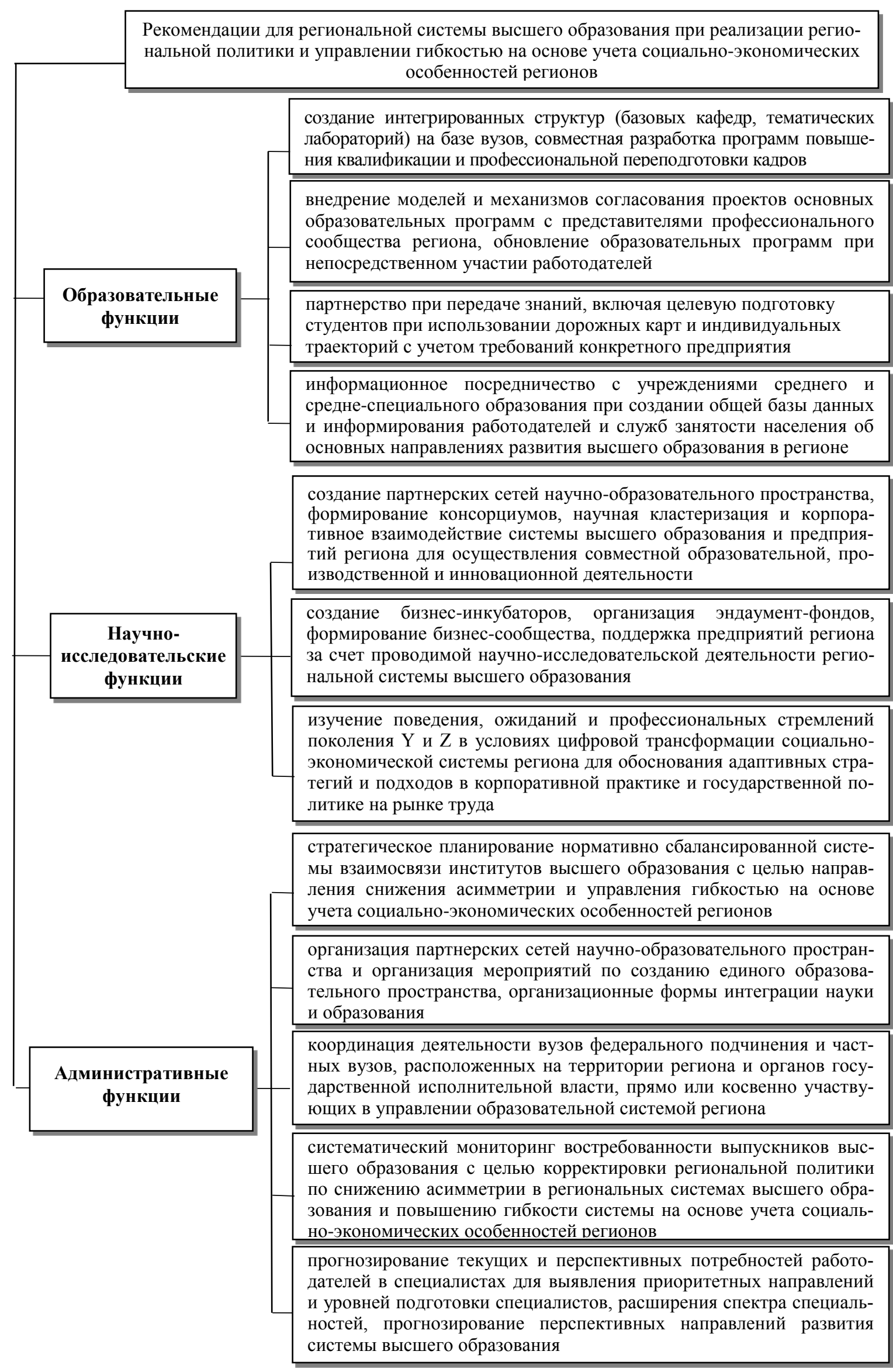

Направления реализации региональной политики на основе учета социально-экономических особенностей регионов

Directions for the implementation of regional policy taking into account the socio-economic characteristics of the regions 


\section{Заключение}

Ожидается, что региональная система высших учебных заведений будет играть ключевую роль в выработке конкретных решений и стратегий, направленных на преодоление динамичных и сложных факторов, подпитывающих кризис устойчивости при реализации региональной политики и управлении гибкостью на основе учета социально-экономических особенностей регионов [Мингалева, 2019]. Чтобы оправдать это ожидание, важно, чтобы образовательные, научно-исследовательские и управленческие функции были легко интегрированы в развитие нормативно сбалансированной системы взаимосвязи институтов высшего образования, которая развивается и усиливается в процессе институционализации [Yarime et al. 2012]. С этой целью использование региональной системы высшего образования в качестве платформы для социально-экономических экспериментов посредством сотрудничества и взаимодействия между академическими кругами, бизнес-сообществом и административным управлением региона позволит внести вклад в моделирование сбалансированного развития региона с учетом его потребностей по принципу «тройной спирали».

\section{Список литературы}

1. Иванова М.В. 2012. Организационные аспекты взаимодействия органов государственной власти в сфере управления высшим профессиональным образованием с вузами региона. [Электронный pecypc]. URL: https://www.researchgate.net/publication/328173317_Organizacionnye_ aspekty_vzaimodejstvia_organov_gosudarstvennoj_vlasti_v_sfere_upravlenia_vyssim_professionalnym_obr azovaniem_s_vuzami_regiona (Дата обращения: 10.11.2020).

2. Макарова Е.Л. 2018. Когнитивное моделирование сбалансированного развития региональных систем высшего образования с учетом потребностей региона. Вестник Пермского университета. Серия «Экономика». 13. 4: 516-531. doi: 10.17072/1994-9960-2018-4-516-531.

3. Мингалева Ж.А. 2019. Соответствие региональной сферы образования задачам формирования профессиональных компетенций и целям устойчивого развития общества (на материалах Пермского края). Региональная экономика и управление: электронный научный журнал. 4. [Электронный ресурс] URL: https://eee-region.ru/article/6018/ (дата обращения: 07.09.2020).

4. Мурашова Е.В., Логинова В.А., Евтушок М.В. 2016. Развитие модели тройной спирали в инновационной экономике: опыт Китая и России. Вестник тихоокеанского государственного университета. 4 (43): 161-170.

5. Огурцова Е.В., Челнокова О.Ю. 2018. Оценка реализации базовых функций региональных систем высшего образования. Известия Саратовского университета. Новая серия. Серия Экономика. Управление. Право, 18 (2): 169-175. doi: 10.18500/1994-2540-2018-18-2-169-175.

6. Сучков Г.В., Ревин И.А. 2012. Сетевое взаимодействие вузов, бизнес-сообщества и социальных институтов в условиях модернизации региональной экономики и системы профессионального образования. Известия высших учебных заведений. Северо-Кавказский регион. Технические науки. 3: 117-122.

7. Федоляк В.С. 2018. Несоответствие рынка образовательных услуг требованиям рынка труда: причины и способы преодоления. Профессиональная ориентация. 2: 49-53.

8. Фирсова А.А., Нархова А.А. 2014. Зарубежные подходы к оценке влияния университета на региональное развитие. Известия Саратовского университета. Новая серия. Серия. Экономика. Управление. Право 14. 2(1): 289-294.

9. Фирсова А.А., Чернышова Г.Ю. 2019. Математические модели для оценки функций систем высшего образования средствами DEA. Известия Саратовского университета. Новая серия. Серия Математика. Механика. Информатика. 19(3): 351-362. doi: 0.18500/1816-9791-2019-19-3-351-362.

10. Cai Y., Etzkowitz H. 2020. Theorizing the Triple Helix model: Past, present, and future. Triple Helix 6(1) :1-38. doi:10.1163/21971927-bja10003.

11. Cai Y., Liu, C. 2020. The Role of University as Institutional Entrepreneur in Regional Innovation System: Towards an Analytical Framework. In: M.T. Preto, A. Daniel, and A. Teixeira, eds. Examining the Role of Entrepreneurial Universities in Regional Development. Hershey. PA: IGI Global. 133-155.

12. Etzkowitz H. 2008. The Triple Helix: University-Industry-Government Innovation in Action. New York/London: Routledge. 
13. Etzkowitz H., Leydesdorff L. 2000. The Dynamics of Innovation: From National Systems and "Mode 2" to a Triple Helix of University-Industry-Government Relations. Research Policy. 29 (2): 109-123. doi: 10.1016/S0048-7333(99)00055-4.

14. Etzkowitz, H. (2008). The Triple Helix: University-Industry-Government Innovation in Action. New York/London: Routledge.

15. Fonseca L., Rodrigues C., Capelleras J-L. 2020. The organizational adaptation of universities to smart specialization: the emergence of strategic network interface units. European Planning Studies. doi: $10.1080 / 09654313.2020 .1854188$

16. Leydesdorff L. 2012. The Triple Helix, Quadruple Helix, ..., and an N-Tuple of Helices: Explanatory Models for Analyzing the Knowledge-Based Economy? Journal of the Knowledge Economy. 3(1): 25-35. doi: 10.1007/s13132-011-0049-4.

17. Morgan K. 1997. The learning region: Institutions, innovation and regional renewal. Regional Studies. 31: 491-503. doi: 10.1080/00343 409750132289.

18. Ogurtsova E., Firsova A., Chelnokova O. 2018. Regional higher education systems and sustainable regional economic development: functional approach. Proceedings of the International Scientific Conference "Competitive, Sustainable and Secure Development of the Regional Economy: Response to Global Challenges" (CSSDRE 2018). Part of series: Advances in Economics, Business and Management Research (AEBMR). 39: 618-622 doi: 10.2991/cssdre-18.2018.126.

19. Powel W.W., Snellman K. 2004. The Knowledge Economy. Annual Review of Sociology 30(1): 199-220 doi: 10.1146/annurev.soc.29.010202.100037.

20. Yarime M., Trencher G., Mino T. et al. 2012. Establishing sustainability science in higher education institutions: towards an integration of academic development, institutionalization, and stakeholder collaborations. Sustain Sci 7: 101-113. https://doi.org/10.1007/s11625-012-0157-5.

\section{References}

1. Ivanova M.V. 2012. Organizational aspects of interaction of government bodies in the sphere of management of higher professional education with universities of the region. URL: https://www.researchgate.net/publication/328173317_Organizacionnye_aspekty_vzaimodejstvia_organov_gos udarstvennoj_vlasti_v_sfere_upravlenia_vyssim_professionalnym_obrazovaniem_s_vuzami_regiona (data obrashcheniya: 10.11.2020). (in Russian)

2. Makarova E.L. 2018. Cognitive modeling of balanced development of regional system of higher education subject to the needs of a region. Perm University Herald. Economy. 13. 4: 516-531. doi: 10.17072/1994-9960-2018-4-516-531. (in Russian)

3. Mingaleva Z.A. 2019. Relevance of the regional sphere of education to the tasks of forming professional competencies and the goals of sustainable development (based on materials from the perm territory). Regional economics and management: electronic scientific journal. 4. URL: https://eeeregion.ru/article/6018/ (data obrashcheniya: 07.09.2020). (in Russian)

4. Murashova E.V., Loginova V.A., Evtushok M.V. 2016. Developing the Triple Helix Model in an Innovation Economy: The Experience of China and Russia. Pacific State University Bulletin. 4 (43): 161-170. (in Russian)

5. Ogurtsova E. V., Chelnokova O. Yu. 2018. Evaluation of realization of basic functions of regional systems of higher education. Izv. Saratov Univ. (N.S.), Ser. Economics. Management. Law. 18 (2): 169-175. doi: 10.18500/1994-2540-2018-18-2-169-175. (in Russian)

6. Suchkov G.V., Revin I.A. 2012. Networking of universities, business community and social institutions in the context of modernization of the regional economy and vocational education system. Proceedings of higher educational institutions. North Caucasian region. Technical science. 3: 117-122. (in Russian)

7. Fedolyak V. S. 2018. Inconsistency of the market of educational services with the requirements of the labor market: reasons and ways of overcoming. Professional orientation. 2: 49-53. (in Russian)

8. Firsova A.A., Narhova A.A. 2014. International Approaches to assessment of university for regional development. Izv. Saratov Univ. (N.S.), Ser. Economics. Management. Law. 14. 2(1): 289-294. (in Russian)

9. Firsova A.A., Chernyshova G.Y. 2019. Mathematical models for evaluation of the higher education system functions with DEA approach. Izv. Saratov Univ. (N.S.), Ser. Math. Mech. Inform. 19 (3): 351-362. doi: 0.18500/1816-9791-2019-19-3-351-362.

10. Cai Y., Etzkowitz H. 2020. Theorizing the Triple Helix model: Past, present, and future. Triple Helix 6 (1) : 1-38. doi:10.1163/21971927-bja10003. 
11. Cai Y., Liu, C. 2020. The Role of University as Institutional Entrepreneur in Regional Innovation System: Towards an Analytical Framework. In: M.T. Preto, A. Daniel, and A. Teixeira, eds. Examining the Role of Entrepreneurial Universities in Regional Development. Hershey. PA: IGI Global. 133-155.

12. Etzkowitz H. 2008. The Triple Helix: University-Industry-Government Innovation in Action. New York/London: Routledge.

13. Etzkowitz H., Leydesdorff L. 2000. The Dynamics of Innovation: From National Systems and "Mode 2" to a Triple Helix of University-Industry-Government Relations. Research Policy. 29 (2): 109-123. doi: 10.1016/S0048-7333(99)00055-4.

14. Etzkowitz, H. (2008). The Triple Helix: University-Industry-Government Innovation in Action. New York/London: Routledge.

15. Fonseca L., Rodrigues C., Capelleras J-L. 2020. The organizational adaptation of universities to smart specialization: the emergence of strategic network interface units. European Planning Studies. doi: $10.1080 / 09654313.2020 .1854188$

16. Leydesdorff L. 2012. The Triple Helix, Quadruple Helix, ..., and an N-Tuple of Helices: Explanatory Models for Analyzing the Knowledge-Based Economy? Journal of the Knowledge Economy. 3(1): 25-35. doi: 10.1007/s13132-011-0049-4.

17. Morgan K. 1997. The learning region: Institutions, innovation and regional renewal. Regional Studies. 31: 491-503. doi: 10.1080/00343 409750132289.

18. Ogurtsova E., Firsova A., Chelnokova O. 2018. Regional higher education systems and sustainable regional economic development: functional approach. Proceedings of the International Scientific Conference "Competitive, Sustainable and Secure Development of the Regional Economy: Response to Global Challenges" (CSSDRE 2018). Part of series: Advances in Economics, Business and Management Research (AEBMR). 39: 618-622 doi: 10.2991/cssdre-18.2018.126.

19. Powel W.W., Snellman K. 2004. The Knowledge Economy. Annual Review of Sociology 30 (1): 199-220 doi: 10.1146/annurev.soc.29.010202.100037.

20. Yarime M., Trencher G., Mino T. et al. 2012. Establishing sustainability science in higher education institutions: towards an integration of academic development, institutionalization, and stakeholder collaborations. Sustain Sci 7: 101-113. https://doi.org/10.1007/s11625-012-0157-5.

\section{ИНФОРМАЦИЯ ОБ АВТОРЕ}

Макарова Елена Львовна,
педагогических нандидат
менеджмента и инновационных технологий
Института управления в экономических,
экологических и социальных системах Южного
федерального университета, г. Ростов-на-Дону,
Россия

\section{INFORMATION ABOUT THE AUTHOR}

Elena L. Makarova, Candidate of Pedagogical Sciences, Associate Professor of Management and Innovative Technologies Department, Institute of Management in Economic, Environmental and Social Systems Southern Federal University, Rostov-on-Don, Russia 